\title{
Pharmacological aspects
}

\section{The AGNP-TDM Expert Group Consensus Guidelines: focus on therapeutic monitoring of antidepressants}

Pierre Baumann, PhD; Sven Ulrich, PhD; Gabriel Eckermann, MD; Manfred Gerlach, PhD; Hans-Joachim Kuss, PhD; Gerd Laux, MD; Bruno Müller-Oerlinghausen, MD; Marie Luise Rao, PhD; Peter Riederer, PhD; Gerald Zernig, MD; Christoph Hiemke, PhD
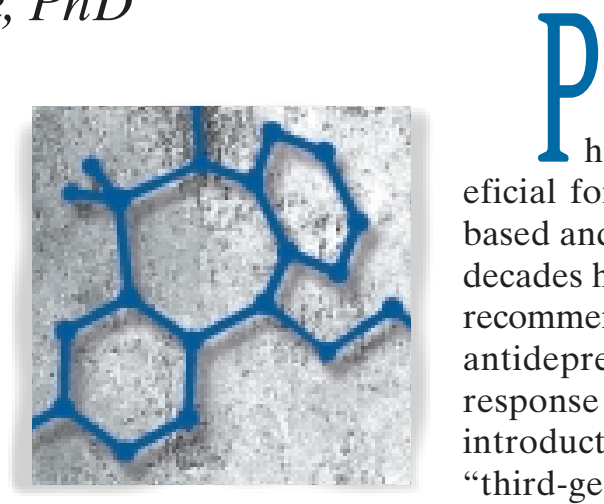
eficial for many patients with depression. Evidencebased and clinical experience collected during the past decades has allowed the introduction of guidelines and recommendations from experts in the field ${ }^{1-3}$ to optimize antidepressant pharmacotherapy. However, partial response and nonresponse are frequent, ${ }^{4}$ despite the introduction of new psychotropic agents, including "third-generation antidepressants," 5 and amelioration and remission rates are still far from optimal. The efficacy of available drugs can be increased, not only by the

Therapeutic drug monitoring (TDM) of psychotropic drugs such as antidepressants has been widely introduced for optimization of pharmacotherapy in psychiatric patients. The interdisciplinary TDM group of the Arbeitsgemeinschaft für Neuropsychopharmakologie und Pharmakopsychiatrie (AGNP) has worked out consensus guidelines with the aim of providing psychiatrists and TDM laboratories with a tool to optimize the use of TDM. Five research-based levels of recommendation were defined with regard to routine monitoring of drug plasma concentrations: (i) strongly recommended; (ii) recommended; (iii) useful; (iv) probably useful; and (v) not recommended. In addition, a list of indications that justify the use of TDM is presented, eg, control of compliance, lack of clinical response or adverse effects at recommended doses, drug interactions, pharmacovigilance programs, presence of a genetic particularity concerning drug metabolism, and children, adolescents, and elderly patients. For some drugs, studies on therapeutic ranges are lacking, but target ranges for clinically relevant plasma concentrations are presented for most drugs, based on pharmacokinetic studies reported in the literature. For many antidepressants, a thorough analysis of the literature on studies dealing with the plasma concentration-clinical effectiveness relationship allowed inclusion of therapeutic ranges of plasma concentrations. In addition, recommendations are made with regard to the combination of pharmacogenetic (phenotyping or genotyping) tests with TDM. Finally, practical instructions are given for the laboratory practitioners and the treating physicians how to use TDM: preparation of TDM, drug analysis, reporting and interpretation of results, and adequate use of information for patient treatment. TDM is a complex process that needs optimal interdisciplinary coordination of a procedure implicating patients, treating physicians, clinical pharmacologists, and clinical laboratory specialists. These consensus guidelines should be helpful for optimizing TDM of antidepressants. 


\section{Pharmacological aspects}

\author{
Selected abbreviations and acronyms \\ CYP cytochrome P-450 \\ GC gas chromatography \\ HPLC high-performance liquid chromatography \\ LOD limit of detection \\ $\mathbf{L O Q} \quad$ limit of quantification \\ PM poor metabolizer \\ SSRI selective serotonin reuptake inhibitor \\ TDM therapeutic drug monitoring \\ UM ultrarapid metabolizer
}

use of augmentation strategies ${ }^{6,7}$ and other combination treatments, ${ }^{8,9}$ but also by analysis of antidepressant drug concentrations in blood plasma. ${ }^{10}$ Recently, a group of psychiatrists, clinical pharmacologists, biochemists, and clinical chemists, all members of the AGNP (Arbeitsgemeinschaft für Neuropsychopharmakologie und Pharmakopsychiatrie; www.agnp.de), worked out consensus guidelines for therapeutic drug monitoring (TDM) in psychiatry, after they had compiled information from the literature. ${ }^{11}$ These guidelines were mainly based on the hypothesis that some inadequate or insufficient treatments of psychiatric patients can be explained by the fact that psychotropic drugs not only differ in their pharmacological profile, but also in their metabolism and pharmacokinetics in the individual patient. Treatment should therefore be adapted according to this situation by using TDM and pharmacogenetic tests. This combined strategy takes into consideration the fact that the fate of the drug depends on both environmental (diet, smoking habits, comorbidities, and comedications) and genetic factors.

Pioneering work in this field was mainly carried out in Sweden, where the first study on the plasma concentration-clinical effectiveness relationship of an antidepressant (nortriptyline) ${ }^{12}$ was performed. This was an outstanding demonstration of the usefulness of the

Keywords: therapeutic drug monitoring; antidepressant; consensus guidelines; pharmacotherapy; psychiatry

Author affiliations: Department of Psychiatry, University of Lausanne, PrillyLausanne, Switzerland (Pierre Baumann, PhD); Department of Clinical Pharmacology, University of Magdeburg, Magdeburg, Germany (Sven Ulrich, $\mathrm{PhD}$ ); Bezirkskrankenhaus Kaufbeuren, Kaufbeuren, Germany (Gabriel Eckermann, MD); Department of Child and Adolescent Psychiatry, University of Wuerzburg, Würzburg, Germany (Manfred Gerlach, PhD); Department of Psychiatry, University of Munich, Munich, Germany (Hans-Joachim Kuss, PhD); Bezirksklinikum Gabersee, Wasserburg/Inn, Germany (Gerd Laux, MD); Drug Commission of the German Medical Association, Berlin and Cologne, Germany (Bruno Müller-Oerlinghausen, MD); Department of Psychiatry, University of Bonn, Bonn, Germany (Marie Luise Rao, PhD); Department of Psychiatry, combination of TDM and pharmacogenetic tests (CYP 2D6) in a pharmacovigilance case situation. ${ }^{13}$ Over the past 20 years, TDM for antidepressants has been widely introduced, but consensus guidelines published to date, or other state-of-the-art reports on the use of TDM for antidepressants concentrated primarily on tricyclic drugs. ${ }^{14-17}$ There is an increasing trend to recommend TDM in combination with pharmacogenetic tests. ${ }^{18,19}$

\section{Aims of the consensus document}

The present consensus guidelines were elaborated to assist psychiatrists, laboratory practitioners, and heads of laboratories involved in psychopharmacotherapy to optimise the use of TDM. Here we focus on antidepressants, * and give recommendations on how to use TDM and genotyping/phenotyping procedures.

\section{Pharmacokinetics, metabolism, and pharmacogenetics of antidepressants}

Antidepressants share many common features, such as high lipophilicity, a molecular weight between 200 and 500 , and basicity. We therefore present a general summary of their pharmacokinetic properties in Table $I^{20-26}$ though numerous compounds constitute exceptions: citalopram is known for its high bioavailability (about $90 \%)$ and relatively low binding to plasma proteins $(80 \%)$; venlafaxine, trazodone, tranylcypromine, and moclobemide display a short (about 2-10 h) and fluoxetine a long plasma half-life (3-15 days, taking into account its active metabolite). It should also be considered that many antidepressants, such as venlafaxine, citalopram,

*This review takes into consideration antidepressant agents currently available in Switzerland and Germany, and therefore does not claim to be exhaustive.

University of Wuerzburg, Würzburg, Germany (Peter Riederer, PhD); Department of Psychiatry, University of Innsbruck, Innsbruck, Austria (Gerald Zernig, MD); Department of Psychiatry, University of Mainz, Germany (Christoph Hiemke, PhD)

Address for correspondence: Prof Pierre Baumann, PhD, Unité de biochimie et psychopharmacologie clinique, Département universitaire de psychiatrie adulte, Centre de neurosciences psychiatriques, $\mathrm{CH}-1008$ Prilly-Lausanne, Switzerland (e-mail: pierre.baumann@chuv.ch)

This article is a modified version of an article published in the journal Pharmacopsychiatry in December 2004: Baumann P, Hiemke C, Ulrich S, et al. The AGNPTDM expert group consensus guidelines: therapeutic drug monitoring in psychiatry. Pharmacopsychiatry. 2004;37:243-265. It is published here with the kind permission of the publishers Georg Thieme Verlag KG, Stuttgart, Germany. 
and mirtazapine, are used as racemic compounds, the enantiomers of which differ in their pharmacological, metabolic, and pharmacokinetic properties. ${ }^{27,28}$

Most antidepressants undergo phase I metabolism by oxidation, such as aromatic ring and aliphatic hydroxylation, $\mathrm{N}$ - and $\mathrm{O}$-dealkylation, $\mathrm{N}$ - and $\mathrm{O}$-oxidation to $\mathrm{N}$-oxides, carbonyl reduction to secondary alcohols, and S-oxidation to sulfoxides or sulfones, which results in an increase in polarity. ${ }^{29}$ The introduction of a functional group (eg, a hydroxy group) or the presence of a tertiary amine group may enable a phase II metabolic step, typically a glucuronidation. ${ }^{30-32}$ Metabolism occurs mainly in the liver and in the intestinal mucosa. It may be age-dependent, and vary as a consequence of the influence of environmental factors, such as somatic diseases, comedication, food, and smoking. TDM should include the assay of active metabolite $^{33-35}$ (eg, clomipramine [norclomipramine] and fluoxetine [norfluoxetine]), but the parent compound/inactive metabolite ratio may be helpful to evaluate the metabolic state or compliance of the patient.

Considerable and clinically relevant knowledge has been acquired during the past 30 years on the important role of cytochrome P-450 (CYP) isozymes, CYP 1A2, CYP 2D6,
CYP 2C9, CYP 2C19, and CYP 3A4/5, in the biotransformation of antidepressants. ${ }^{36-42}$ The genetically determined polymorphism of CYP 2D6 is of high clinical relevance for antidepressants, which are substrates of this isozyme, including tricyclic antidepressants, some selective serotonin reuptake inhibitors (SSRIs) (eg, paroxetine and fluoxetine), and "third-generation" antidepressants (eg, venlafaxine and mirtazapine). About $5 \%$ to $8 \%$ and $1 \%$ to $7 \%$ of the Caucasian population are considered as poor metabolizers (PMs) or ultrarapid metabolizers (UMs), respectively (Table I). . $22,43,44$ In Caucasians, there is a lower proportion (3\%-5\%) of PMs of CYP 2C19, which is frequently involved in $\mathrm{N}$-demethylation of tertiary amines (amitriptyline and citalopram). CYP 3A4/5 shows wide interindividual variability in its activity. CYP $3 \mathrm{~A} 5$ is expressed in only one-third of the Caucasian population. ${ }^{45}$ As regards CYP 1A2, only its inducibility (eg, by tobacco smoke) is genetically polymorphic. ${ }^{46,47}$ Clinically, a PM status may represent a higher risk for adverse effects in patients treated with antidepressants known to be substrates of the deficient enzyme, while UMs undergo a higher risk for nonresponse, due to subtherapeutic plasma concentrations. ${ }^{39,48-53}$ The clinical relevance of the genetic

\begin{tabular}{|c|c|c|}
\hline Pharmacokinetic phase & & Characteristics \\
\hline \multirow[t]{2}{*}{ Absorption } & A & Good absorption from gastrointestinal tract \\
\hline & & Maximum plasma concentration within a short time after administration ( $t_{\max }$ of about 0.5 to $4 \mathrm{~h}$ ) \\
\hline \multirow[t]{6}{*}{ Distribution } & D & High distribution volume \\
\hline & & Fast distribution from plasma to the central nervous system \\
\hline & & 10 to 40 times higher levels in brain than in blood \\
\hline & & Possible regulation of transport intestine-blood and blood-brain by transport proteins (P-glycoprotein) \\
\hline & & Low plasma concentrations in steady-state conditions (trough levels: $0.5-500 \mathrm{ng} / \mathrm{mL}$ ) \\
\hline & & High plasma protein binding (90\%-99\%) \\
\hline \multirow[t]{9}{*}{ Metabolism } & M & Metabolism: a prerequisite for excretion \\
\hline & & High first-pass metabolism (systemic availability: 10\%-70\%) \\
\hline & & Main metabolic enzyme systems: cytochrome P-450, UDP-glucuronosyltransferases \\
\hline & & Genetic polymorphisms for some enzymes (extensive, intermediate, poor, and ultrarapid metabolizers) \\
\hline & & Inducibility of some enzymes by drugs or other xenobiotics \\
\hline & & Generally formation of active, but more polar metabolites \\
\hline & & Occurrence and relevance of metabolism in brain doubtful \\
\hline & & Important effect of hepatic insufficiency on hepatic elimination \\
\hline & & High risk for inhibition of drug metabolism by comedication, inhibitors of cytochrome P-450 \\
\hline \multirow[t]{4}{*}{ Elimination } & E & Low renal excretion \\
\hline & & Small effect of renal insufficiency on plasma kinetics of drug and its metabolites \\
\hline & & Slow elimination from plasma (half-life $12-36 \mathrm{~h}$ ), mainly by hepatic metabolism \\
\hline & ADME & Linear pharmacokinetics at clinically relevant doses \\
\hline
\end{tabular}

Table I. General pharmacokinetic properties (absorption, distribution, metabolism, and elimination [ADME]) of antidepressants. ${ }^{11,20}$ 


\section{Pharmacological aspects}

polymorphisms of UDP-glucuronosyltransferases in pharmacopsychiatry is not clear. ${ }^{30,54}$

Genotyping, which represents a "trait marker," is readily available and clinically recommended for CYP 1A2, CYP 2C9, CYP 2C19, CYP 2D6, and CYP 3A4/5; phenotyping, used as a "state-marker," may be performed for the same enzymes. The result of genotyping is not influenced by environmental factors and has life-long validity. Phenotyping requires the administration of drugs and is therefore a more invasive procedure. Therefore, indications for phenotyping and genotyping may differ.

As mentioned in Table I, transport proteins such as P-glycoprotein in the intestinal mucosa and in the blood-brain barrier may be implicated in the regulation of the availability of antidepressants for the brain, but there is still a lack of clinical data. ${ }^{55-57}$

\section{Relationships between drug doses, plasma concentrations, and clinical variables}

TDM is based on the hypothesis assumption that there is a well-defined relationship between the drug plasma concentration and its clinical effects (therapeutic effect, adverse effects, and toxicity). However, while such a relationship is generally well admitted for lithium and for the tricyclic antidepressants nortriptyline, amitriptyline, desipramine, and imipramine, inconsistent results were obtained in studies on other tricyclic or similarly structured antidepressants, SSRIs, and other recently introduced antidepressants. ${ }^{20,58-62}$ Interestingly, systematic reviews and meta-analyse ${ }^{14,59}$ that were based on adequately designed studies yielded evidence of a relationship between clinical variables and plasma concentration for some tricyclic drugs. This suggests that numerous studies were poorly designed methodologically in order to demonstrate an evident relationship between concentration and effects or side effects. Recently, Ulrich and Läuter ${ }^{60}$ defined criteria for quality assessment of TDM studies, which include the use of valid chemical and analytical methods, adequate psychopathology rating scales, appropriate selection criteria for patients (eg, exclusion of known nonresponders), and reporting of comedication.

\section{Analytical procedures}

Plasma or serum samples are generally used for TDM. Concentrations of antidepressants are low, most often in the $\mathrm{nmol} / \mathrm{L}(\mathrm{ng} / \mathrm{mL})$ range. Therefore, highly sensitive and selective analytical methods are needed for accurate and precise quantification. ${ }^{63-66}$

Most laboratories use now gas chromatography (GC) or high-performance liquid chromatography (HPLC) for the assay of antidepressants for TDM purposes. For GC, the most recommended detection systems are mass spectrometry (GC-MS) or nitrogen phosphor detectors (GC-NPD). Ultraviolet (UV) detectors, fluorescence detectors, and mass spectrometry (LC-MS), in increasing order, are useful for a selective and sensitive drug assay. Clearly, the need for sample preparation before chromatographic separation represents a time-consuming step, and this procedure also implies a limited sample throughput, despite the availability of automated sample preparation prior to GC or HPLC. ${ }^{67}$ Direct injection ("column switching HPLC") of plasma or serum into the HPLC system is now available for a number of antidepressants. ${ }^{6870}$ LC-MS and LC-MS-MS (tandem mass spectrometry) will increasingly be the method of choice, as it may be applied to almost any psychotropic drug including metabolites, while GC-MS is applicable only for volatile compounds.

\section{Economic aspects of TDM in psychiatry}

TDM for a single psychoactive drug, including a metabolite, costs between 20 and $80 €$, which includes costs for staff, instrumentation, chemicals, and other materials. In some countries, analyses may be billed according to the analytical technique used (higher rates for mass spectrometric quantification).

A proof of cost-effectiveness has been provided for only a few antidepressants. ${ }^{71,72}$ However, additional studies are required. They should be designed to take account of the complexity of the TDM process (Figure 1). For example, a recent prospective study carried out under naturalistic conditions showed that dose adjustment by the treating physician was frequently inappropriate, in that he or she neglected the results of the laboratory assays. ${ }^{73}$

Preliminary data suggest that phenotyping or genotyping of patients may help decrease the cost of their treatment with substrates of CYP 2D6. ${ }^{74}$ The costs of treating patients who are either UMs or PMs (CYP 2D6) are seemingly thousands of US dollars per year higher than those for extensive metabolizers (EMs). ${ }^{75}$ However, the tools to assess the cost-effectiveness of pharmacogenetic tests are still insufficiently developed. ${ }^{76}$ 


\section{Consensus}

TDM should be limited to situations where it may be expected that the result will help to solve a therapeutic problem. There are many indications for using TDM (Table II) in antidepressant pharmacotherapy, such as suspicion of noncompliance or intoxication. In pharmacovigilance programs, TDM may be considered as a valid indication for all drugs and groups of patients. To recommend TDM as routine monitoring, it must be proven that TDM is of value. Five levels of recommendation for TDM were defined, which range from "strongly recommended" to "not recommended." In a second step, a recommendation tailored to the individual drug was defined.

\section{Levels of recommendations to use TDM as routine monitoring}

The therapeutic strategy will only be improved by the use of TDM, if the already mentioned criteria are fulfilled. ${ }^{60}$ There is sufficient evidence that TDM can be useful for patients treated with antidepressants, as concluded by the authors of this consensus guideline, after a careful examination of the literature: (i) guidelines; (ii) meta-analyses; (iii) prospective studies on the clinical effectiveness of drugs in which drug plasma concentrations were reported; and (iv) pharmacokinetic studies. However, the latter often do not allow definition of a therapeutic plasma concentration range, in the absence of clinical data. Five levels of recommendation to use

\begin{tabular}{|l|l|l|l}
\multicolumn{1}{|c}{ Routine monitoring } & \multicolumn{1}{c}{ Specific indication } \\
$\begin{array}{l}\text { Dose titration after start or change of medication, } \\
\text { relapse prevention }\end{array}$ & $\begin{array}{l}\text { Lack of response, insufficient response, side effects at } \\
\text { therapeutic doses, potential drug interaction or other } \\
\text { specific indications (Table } I \text { ) }\end{array}$ \\
Blood sample collection
\end{tabular}

Steady state at the time of minimal drug concentration (trough level)

Complete request form (demographic data, diagnosis, medication, comedication, therapeutic effect, and side effects)

\section{Laboratory measurements}

Use of validated methods (linearity, accuracy, precision, sensitivity, specificity)

Internal and external quality control management

\section{Communication of results}

Reporting of drug concentration including unit and range of target concentration Interpretation related to the patient's state

\section{Therapeutic management}

Consideration of the patient's clinical state

Consideration of drug concentration in plasma and interpretation of results

\section{Optimization of drug treatment}

Change in dose, cessation of drug, change in comedication, discussion of the results with the patient (eg, compliance, safety), pharmacogenetic monitoring, no intervention

Figure 1. Summary of the therapeutic drug monitoring (TDM) process for optimization of the pharmacotherpy of psychiatric patients. Routine monitoring should be restricted to psychoactive drugs with established therapeutic ranges and who levels of recommendation to use TDM are at least 2 (Table IV). Specific requests may be useful for any psychoactive drug and many indications (Table I), even without well-established therapeutic ranges." 


\section{Pharmacological aspects}

TDM as routine monitoring were defined as follows, as reported earlier. ${ }^{10}$

\section{Strongly recommended}

- Established therapeutic range

- Level of evidence: Controlled clinical trials have shown benefit of TDM; reports on toxic effects at "supratherapeutic" plasma concentrations.

- Clinical consequences: At therapeutic plasma concentrations highest probability of response; at "subtherapeutic" plasma concentrations response rate similar to placebo; at plasma concentrations higher than therapeutic concentrations increasing risk of adverse effects.

\section{Recommended}

- Suggested therapeutic ranges obtained from plasma concentrations at therapeutically effective doses (fixed dose studies).

- Level of evidence: At least one well-designed prospective study with well-defined outcome criteria reports intoxications at "supratherapeutic" plasma concentrations.

- Clinical consequences: TDM most probably will optimize response in nonresponders: at "subtherapeutic" plasma concentrations risk of poor response; at "supratherapeutic" plasma concentrations risk of adverse effects and/or decreased response.

\section{Useful}

- Suggested therapeutic ranges are plasma concentrations at effective doses obtained from steady-state pharmacokinetic studies.

- Level of evidence: Clinical data from retrospective analysis of TDM data; single case reports; or nonsystematic clinical experience.

- Clinical consequences:TDM useful to control whether plasma concentrations are plausible for a given dose; optimizing of clinical response in nonresponders who display low concentrations is possible.

\section{Probably useful}

- Suggested therapeutic ranges from steady-state pharmacokinetic studies at therapeutically effective doses.

- Level of evidence: Valid clinical data so far lacking or inconsistent results.
- Clinical consequences:TDM useful to control whether plasma concentrations are plausible for a given dose.

\section{Not recommended}

- Unique pharmacology of the drug, eg, irreversible blockade of an enzyme or flexible dosing according to clinical symptoms.

- Level of evidence: Textbook knowledge, basic pharmacology.

- Clinical consequences: TDM should not be used.

\section{Drug-specific TDM recommendations}

The knowledge of plasma concentrations ranges observed after treatment of subjects at well-defined doses of the antidepressant (Table III) may efficiently help the clinician in some of the situations listed in Table II: suspicion of noncompliance, drug interactions, problems occurring after switching from an original preparation to a generic form (and vice versa), or presence of a pharmacogenetic PM or UM status. The information available in Table III is also helpful in situations where the levels of recommendations 3 and 4 apply (ie, TDM useful or probably useful).

Suspected noncompliance
Drugs, for which TDM is mandatory for safety reasons (eg, lithium)
Lack of clinical response, or insufficient response, even if dose is
considered as adequate
Adverse effects, despite the use of generally recommended doses
Suspected drug interactions
TDM in pharmacovigilance programs
Combination treatment with a drug known for its interaction
potential, in situations of comorbidities, "augmentation," etc
Relapse prevention in long-term treatments, prophylactic treat-
ments
Recurrence despite good compliance and adequate doses
Presence of a genetic particularity concerning the drug metabolism
(genetic deficiency, gene multiplication)
Children and adolescents
Elderly patients (>65 years)
Patients with pharmacokinetically relevant comorbidities (hepatic
or renal insufficiency, cardiovascular disease)
Forensic psychiatry
Problems occurring after switching from an original preparation to
a generic form (and vice versa)

Table II. General indications for therapeutic drug monitoring (TDM) of antidepressants. ${ }^{11}$ 


\begin{tabular}{|c|c|c|c|c|c|}
\hline \multirow[t]{2}{*}{ Antidepressant } & Active & \multicolumn{4}{|c|}{ Dose-related steady-state plasma concentrations* } \\
\hline & $\begin{array}{l}\text { metabolite } \\
\text { (or metabolite } \\
\text { recommended } \\
\text { for TDM) }\end{array}$ & $\begin{array}{c}\text { Dose } \\
\text { (mg/day) }\end{array}$ & $\begin{array}{l}\text { Parent compound } \\
\text { (ng/mL) }\end{array}$ & $\begin{array}{l}\text { Metabolite } \\
\text { (ng/mL) }\end{array}$ & References \\
\hline \multirow[t]{5}{*}{ Amitriptyline } & Nortriptyline & 150 & $102 \pm 59(34-278)$ & $85 \pm 60(16-326)$ & Baumann et al, ${ }^{77} 1986$ \\
\hline & & 150 & $122 \pm 62$ & $84 \pm 48$ & Jungkunz and Kuss, 1980 \\
\hline & & 150 & $76 \pm 30$ & $84 \pm 38$ & Breyer-Pfaff et al, ${ }^{79} 1982$ \\
\hline & & 150 & $100 \pm 41$ & $71 \pm 38$ & Breyer-Pfaff et al, ${ }^{80} 1982$ \\
\hline & & 200 & $146 \pm 21$ (sem) & $129 \pm 23$ (sem) & Kupfer et al, ${ }^{81} 1977$ \\
\hline \multirow[t]{2}{*}{ Citalopram } & Demethylcitalopram & 40 & $86 \pm 38$ & $35 \pm 11$ & Baumann et al, 821996 \\
\hline & & 40 iv & $70 \pm 23$ & $30 \pm 12$ & Baumann et al, $_{1}^{83} 1998$ \\
\hline \multirow[t]{7}{*}{ Clomipramine } & Demethylclomipramine & 75 bid & $63 \mathrm{md}(22-230) *$ & $148 \mathrm{md}(51-331) *$ & Kramer Nielsen et al, ${ }^{84} 1992$ \\
\hline & & 50 & $24 \mathrm{md}(5-69)^{*}$ & $15 \mathrm{md}(6-78)^{*}$ & DUAG, ${ }^{85} 1999$ \\
\hline & & 75 & $38 \mathrm{md}(9-78)^{*}$ & $43 \mathrm{md}(5-102)^{*}$ & DUAG, ${ }^{85} 1999$ \\
\hline & & 125 & $83 \mathrm{md}(31-224) *$ & $105 \mathrm{md}(41-335)^{*}$ & DUAG, ${ }^{85} 1999$ \\
\hline & & 200 & $202 \mathrm{md}(50-340)$ * & $283 \mathrm{md}(138-446)^{*}$ & DUAG, ${ }^{85} 1999$ \\
\hline & & $100 \mathrm{iv}$ & $122 \pm 73$ & $145 \pm 118$ & $\begin{array}{l}\text { Müller-Oerlinghausen and } \\
\text { Fähndrich, }{ }^{86} 1985\end{array}$ \\
\hline & & 150 & $74-310$ & $69-267$ & Burch et $a l_{1}^{87} 1982$ \\
\hline \multirow[t]{3}{*}{ Desipramine } & & 200 & $173(28-882)$ & & Friedel et al, ${ }^{88} 1979$ \\
\hline & & $186 \pm 24$ & $188 \pm 152$ & & Amsterdam et al, ${ }^{89} 1985$ \\
\hline & & $75-250$ & $16-502$ & & Nelson et al, ${ }^{90} 1985$ \\
\hline \multirow[t]{4}{*}{ Dothiepine } & Dothiepine-SO & 150 & $95 \pm 67$ & $323 \pm 191$ & Maguire et al, ${ }^{91} 1982$ \\
\hline & Northiaden & 150 & & $16 \pm 12$ & Maguire et al, ${ }^{91} 1982$ \\
\hline & Dothiepine-SO & $3.22 \pm 0.99 \mathrm{mg} / \mathrm{kg}$ & $67(4-258)$ & $352(45-953)$ & Ilett et al, ${ }^{92} 1993$ \\
\hline & Northiaden & $3.22 \pm 0.99 \mathrm{mg} / \mathrm{kg}$ & & $37(0-230)$ & Ilett et al, ${ }^{92} 1993$ \\
\hline \multirow[t]{5}{*}{ Doxepin (DOX) } & Demethyldoxepin (DDOX) & ) $\quad 250$ & $484 \pm 251 \mathrm{nmol} / \mathrm{L}^{\dagger}$ & & Adler et al, ${ }^{93} 1997$ \\
\hline & Demethyldoxepin & 250 & $130 \pm 113$ & $132 \pm 94$ & Deuschle et al, ${ }^{94} 1997$ \\
\hline & trans-Demethyldoxepin & 250 & & $72 \pm 60$ & Deuschle et al, ${ }^{94} 1997$ \\
\hline & cis-Demethyldoxepin & 250 & & $60 \pm 45$ & Deuschle et al, ${ }^{94} 1997$ \\
\hline & & $143 \pm 30$ & $89 \pm 75^{+}$ & & Leucht et al, ${ }^{95} 2001$ \\
\hline \multirow[t]{2}{*}{ Escitalopram ${ }^{\ddagger}$} & S-Demethylcitalopram & $10^{\ddagger}$ & $27 \pm 14$ & $14 \pm 5$ & Bondolfi et al, ${ }^{96} 1996$ \\
\hline & & $10^{\ddagger}$ & $28 \pm 9$ & $11 \pm 3$ & Bondolfi et al, ${ }^{97} 2000$ \\
\hline \multirow[t]{3}{*}{ Fluoxetine } & Norfluoxetine & 20 & $80(9-265) \mathrm{md}$ & $126(30-300) \mathrm{md}$ & Lundmark et al, $^{98} 2001$ \\
\hline & & 40 & $195(40-496) \mathrm{md}$ & $221(20-449) \mathrm{md}$ & \\
\hline & & 20 & $97 \pm 51$ & $128 \pm 49$ & Amsterdam et al, ${ }^{99} 1997$ \\
\hline \multirow[t]{6}{*}{ Fluvoxamine } & & 100 & $90 \pm 29(f)$ & & Härtter et al, ${ }^{100} 1998$ \\
\hline & & 100 & $59 \pm 22(m)$ & & Härtter et al, ${ }^{100} 1998$ \\
\hline & & 200 & $274 \pm 73(f)$ & & Härtter et al, ${ }^{100} 1998$ \\
\hline & & 200 & $237 \pm 90(m)$ & & Härtter et al, ${ }^{100} 1998$ \\
\hline & & $229 \pm 47$ & $142 \pm 108(20-417)$ & & Kasper et al, ${ }^{101} 1993$ \\
\hline & & 200 & $162 \pm 144(13-833)$ & & Gerstenberg et al, ${ }^{102} 2003$ \\
\hline
\end{tabular}

Table III. Dose-related steady-state plasma concentrations of antidepressants. ${ }^{11}$ Generally, arithmetic means \pm standarad deviations are given; numbers in parentheses indicate ranges. md, median value; gm, geometric mean; $m$, males; $f$, females. * Extensive metabolizers (CYP 2D6). ${ }^{+}$Doxepin + desmethyldoxepin. ${ }^{*}$ Patients were treated with $20 \mathrm{mg} /$ day citalopram, and S-citalopram and its metabolite were measured. ${ }^{5}$ Nonsmokers. "Smokers. "Concentrations given in ng.kg/mL.mg, in extensive metabolizers (CYP 2D6). "Concentrations show very little differences when given $50 \mathrm{mg} /$ day tid. 


\section{Pharmacological aspects}

\begin{tabular}{|c|c|c|c|c|c|}
\hline \multirow[t]{2}{*}{ Antidepressant } & Active & \multicolumn{4}{|c|}{ Dose-related steady-state plasma concentrations* } \\
\hline & $\begin{array}{l}\text { metabolite } \\
\text { (or metabolite } \\
\text { recommended } \\
\text { for TDM) }\end{array}$ & $\begin{array}{c}\text { Dose } \\
\text { (mg/day) }\end{array}$ & $\begin{array}{l}\text { Parent compound } \\
\text { (ng/mL) }\end{array}$ & $\begin{array}{l}\text { Metabolite } \\
\text { (ng/mL) }\end{array}$ & References \\
\hline Imipramine & Desipramine & 225 & $(6-268)$ & $(18-496)$ & Reisby et al, 1031977 \\
\hline \multirow[t]{2}{*}{ Maprotiline } & (Desmethylmaprotiline) & 150 & $116 \pm 47$ & & Gabris et al, ${ }^{104} 1985$ \\
\hline & & $236 \pm 32$ & $202 \pm 134(12-428)$ & & Kasper et al, ${ }^{101} 1993$ \\
\hline Mianserin & Demethylmianserin & 30 & $22(12-48)$ & $9(3-24)$ & Otani et al, 1051991 \\
\hline \multirow[t]{4}{*}{$(\mathrm{MIA})$} & (DMIA) & 30 & $14(6-37)(S-M I A)$ & & Mihara et al, ${ }^{106} 1997$ \\
\hline & & 30 & 9 (4-18) (R-MIA) & & Mihara et al, ${ }^{106} 1997$ \\
\hline & & 60 & $37 \pm 19(14-67)(S-M I A)$ & $10 \pm 5(6-23)(S-D M I A)$ & Eap et al, $^{107} 1999$ \\
\hline & & 60 & $19 \pm 11(10-51)(R-\mathrm{MIA})$ & $21 \pm 15(10-52)(R-\mathrm{DMIA})$ & Eap et al, ${ }^{107} 1999$ \\
\hline \multirow[t]{5}{*}{ Mirtazapine } & (Demethylmirtazapine) & 15 & $7.3 \pm 3.2$ & & Timmer et al, $^{108} 1995$ \\
\hline & & 30 & $18 \pm 7$ & & Timmer et al, ${ }^{108} 1995$ \\
\hline & & 45 & $28 \pm 12$ & & Timmer et al, ${ }^{108} 1995$ \\
\hline & & 60 & $38 \pm 16$ & & Timmer et al, ${ }^{108} 1995$ \\
\hline & & 70 & $46 \pm 16$ & & Timmer et al, ${ }^{108} 1995$ \\
\hline Moclobemide & & 100 tid & $216 \pm 55$ & & Schoerlin et al, ${ }^{109} 1987$ \\
\hline \multirow[t]{2}{*}{ Nortriptyline } & & 150 & $141 \pm 48(48-238)$ & & \\
\hline & & $75-225$ & $90 \pm 40(32-164)$ & & Asberg et al, ${ }^{12} 1971$ \\
\hline \multirow[t]{3}{*}{ Paroxetine } & & 30 & $36.3(1.7-60.8)$ & & Lundmark et al, ${ }^{110} 1989$ \\
\hline & & 30 & $27 \mathrm{md}(12-45)^{*}$ & & Sindrup et al, ${ }^{111} 1992$ \\
\hline & & 30 & $36(9-70)$ & & Kaye et al, ${ }^{112} 1989$ \\
\hline Reboxetine & & 4 & $50 \pm 20$ & & Pellizzoni et al, ${ }^{113} 1996$ \\
\hline \multirow[t]{5}{*}{ Sertraline } & (Norsertraline) & 50 & $12 \pm 17$ gm (3-134) & $30 \pm 24$ gm (7-143) & Lundmark et al, ${ }_{1}^{114} 2000$ \\
\hline & & 100 & $19 \pm 18$ gm (3-109) & $45 \pm 35$ gm (10-273) & Lundmark et al, ${ }^{114} 2000$ \\
\hline & & 150 & $31 \pm 29$ gm (8-145) & $65 \pm 47$ gm (7-138) & Lundmark et al, ${ }^{114} 2000$ \\
\hline & & 200 & $29 \pm 18$ gm (9-82) & $87 \pm 43$ gm (40-189) & Lundmark et al, ${ }^{114} 2000$ \\
\hline & & 50 & $12 \pm 8(4-32)$ & & Axelson et al, ${ }^{115} 2002$ \\
\hline \multirow[t]{3}{*}{ Trazodone } & m-Chlorophenylpiperazine & 150 & $624(271-1062)$ & 65 (34-108) & Otani et al, ${ }^{116} 1998$ \\
\hline & & 150 & $680 \pm 257^{\S}$ & $65 \pm 21^{\varsigma}$ & Mihara et al, ${ }^{117} 2001$ \\
\hline & & 150 & $541 \pm 277^{\| \prime}$ & $56 \pm 21^{11}$ & Mihara et al, ${ }^{117} 2001$ \\
\hline \multirow{3}{*}{$\begin{array}{l}\text { Trimipramine } \\
\text { (TRI) }\end{array}$} & Desmethyltrimipramine & 200 & $277 \pm 67$ & $169 \pm 51$ & Cournoyer et al, 1987 \\
\hline & (DTRI) & & $21 \pm 11(7-47)(\mathrm{L}-\mathrm{TRI})^{n}$ & $7 \pm 6(1-23)(L-D T R I)^{n}$ & Eap et al, ${ }^{119} 2000$ \\
\hline & & & $18 \pm 6(8-32)(D-T R I)^{n}$ & $10 \pm 7(2-29)(D-D T R I)^{n}$ & Eap et al, ${ }^{119} 2000$ \\
\hline \multirow[t]{5}{*}{ Venlafaxine } & O-Demethylvenlafaxine & 75 bid $^{\#}$ & $56 \pm 31$ & $194 \pm 75$ & Troy et al, 1920 \\
\hline & & 75 & $75 \pm 93(5-427)$ & $116 \pm 65(16-260)$ & Reis et al, ${ }^{121} 2002$ \\
\hline & & 150 & $109 \pm 232(4-1903)$ & $186 \pm 94(16-411)$ & Reis et al, ${ }^{121} 2002$ \\
\hline & & 225 & $178 \pm 283(9-1421)$ & $232 \pm 132(63-736)$ & Reis et al, ${ }_{121}^{121} 2002$ \\
\hline & & 300 & $155 \pm 109(21-438)$ & $249 \pm 121(104-516)$ & Reis et al, ${ }_{1}^{121} 2002$ \\
\hline Viloxazine & & 300 & 1200 (ca 400-1600) & & $\begin{array}{l}\text { Müller-Oerlinghausen and } \\
\text { Ruether, } 1221979\end{array}$ \\
\hline
\end{tabular}

Table III. Continued. 


\begin{tabular}{|c|c|c|c|c|}
\hline $\begin{array}{l}\text { Drug and active } \\
\text { metabolite for } \\
\text { antidepressants }\end{array}$ & $\begin{array}{l}\text { Recommended } \\
\text { therapeutic range } \\
\text { (consensus) }\end{array}$ & $\begin{array}{l}\text { Level of } \\
\text { recommendation }\end{array}$ & $\begin{array}{l}\text { References for reports on } \\
\text { therapeutic ranges }\end{array}$ & $\begin{array}{l}\text { Reference for reports on } \\
\text { intoxications }\end{array}$ \\
\hline $\begin{array}{l}\text { Amitriptyline plus } \\
\text { nortriptyline }\end{array}$ & $80-200 \mathrm{ng} / \mathrm{mL}$ & 1 & $\begin{array}{l}\text { Ulrich and Läuter, }{ }^{60} 2002 \\
\text { Pedersen et al, }{ }^{123} 1982\end{array}$ & Preskorn and Jerkovich, 1924 \\
\hline Citalopram & $30-130$ ng/mL & 3 & $\begin{array}{l}\text { Bjerkenstedt et al, }{ }_{1}^{125} 1985 \\
\text { Leinonen et al, }{ }^{126} 1996\end{array}$ & Jonasson and Saldeen, ${ }^{127} 2002$ \\
\hline $\begin{array}{l}\text { Clomipramine plus } \\
\text { norclomipramine }\end{array}$ & $175-450 \mathrm{ng} / \mathrm{mL}$ & 1 & $\begin{array}{l}\text { DUAG, }^{85} 1999 \\
\text { Gex-Fabry et al, }{ }^{128} 1999 \\
\text { Mavissakalian et al, }{ }^{129} 1990\end{array}$ & Mclntyre et al, ${ }^{130} 1994$ \\
\hline Desipramine & $100-300 \mathrm{ng} / \mathrm{mL}$ & 2 & $\begin{array}{l}\text { Perry et al, }{ }^{59} 1994 \\
\text { Pedersen et } \text { al, }^{123} 1982\end{array}$ & Preskorn and Jerkovich, 1924 \\
\hline Doxepin plus nordoxepin & $50-150 \mathrm{ng} / \mathrm{mL}$ & 3 & $\begin{array}{l}\text { Leucht et al, }{ }^{95} 2001 \\
\text { Rodriguez de la Torre et al, }{ }^{131} 2001\end{array}$ & Preskorn and Fast, ${ }^{132} 1992$ \\
\hline Escitalopram & $15-80 \mathrm{ng} / \mathrm{mL}$ & 4 & SPC & \\
\hline Fluoxetine plus norfluoxetine & $120-300 \mathrm{ng} / \mathrm{mL}$ & 3 & $\begin{array}{l}\text { Lundmark et al, }{ }^{98} 2001 \\
\text { Amsterdam et al, }{ }^{99} 1997\end{array}$ & \\
\hline Fluvoxamine & $150-300 \mathrm{ng} / \mathrm{mL}$ & 4 & 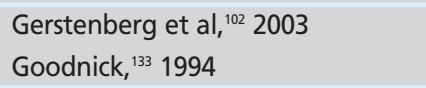 & Kasper et al, ${ }^{101} 1993$ \\
\hline $\begin{array}{l}\text { Imipramine plus } \\
\text { desipramine }\end{array}$ & $175-300 \mathrm{ng} / \mathrm{mL}$ & 1 & Perry et al, ${ }^{59} 1994$ & Pedersen et al, ${ }^{123} 1982$ \\
\hline Maprotilin & $125-200 \mathrm{ng} / \mathrm{mL}$ & 3 & SPC, Kasper et al, ${ }^{101} 1993$ & Pedersen et al, ${ }^{123} 1982$ \\
\hline Mianserin & $15-70 \mathrm{ng} / \mathrm{mL}$ & 3 & Montgomery et al, ${ }^{134} 1978$ & Isacsson et al, ${ }^{135} 1997$ \\
\hline Mirtazapine & $40-80 \mathrm{ng} / \mathrm{mL}$ & 3 & Timmer et al, ${ }^{136} 2000$ & Velazquez et al,,137 2001 \\
\hline Moclobemide & $300-1000 \mathrm{ng} / \mathrm{mL}$ & 4 & $\begin{array}{l}\text { Fritze et al, }{ }^{138} 1989 \\
\text { Gex-Fabry et al, }{ }^{139} 1995\end{array}$ & Hernandez et al, ${ }^{140} 1995$ \\
\hline Nortriptyline & $70-170 \mathrm{ng} / \mathrm{mL}$ & 1 & $\begin{array}{l}\text { Perry et al, }{ }^{12} 1994 \\
\text { Åsberg et } \text { al, }^{59} 1971\end{array}$ & Åsberg et al, ${ }^{141} 1970$ \\
\hline Paroxetine & $70-120 \mathrm{ng} / \mathrm{mL}$ & 3 & $\begin{array}{l}\text { Lundmark et al, }{ }^{114} 2000 \\
\text { Tasker et al, }{ }^{142} 1989\end{array}$ & \\
\hline Reboxetine & $10-100 \mathrm{ng} / \mathrm{mL}$ & 4 & Ohman et al, ${ }^{143} 2001$ & \\
\hline Sertraline & $10-50 \mathrm{ng} / \mathrm{mL}$ & 3 & Lundmark et al, ${ }_{114}^{114} 2000$ & Milner et al, ${ }^{144} 1998$ \\
\hline Tranylcypromine & $0-50 \mathrm{ng} / \mathrm{mL}$ & 5 & Burke and Preskorn, 1451999 & Iwersen and Schmoldt, ${ }^{146} 1996$ \\
\hline Trazodone & $650-1500 \mathrm{ng} / \mathrm{mL}$ & 3 & $\begin{array}{l}\text { Monteleone et al, }{ }^{147} 1989 \\
\text { Goeringer et al, }{ }^{148} 2000\end{array}$ & \\
\hline Trimipramine & $150-350 \mathrm{ng} / \mathrm{mL}$ & 3 & $\begin{array}{l}\text { Cournoyer et al, }{ }^{118} 1987 \\
\text { Isaccson et al, }{ }^{135} 1997\end{array}$ & \\
\hline $\begin{array}{l}\text { Venlafaxine plus } \\
\text { O-desmethylvenlafaxine }\end{array}$ & $195-400 \mathrm{ng} / \mathrm{mL}$ & 2 & $\begin{array}{l}\text { Veefkind et al, }{ }^{149} 2000 \\
\text { Levine et al, }{ }^{150} 1996\end{array}$ & \\
\hline Viloxazine & $20-500 \mathrm{ng} / \mathrm{mL}$ & 3 & $\begin{array}{l}\text { Norman et al, }{ }^{151} 1980 \\
\text { Altamura et al, }{ }^{152} 1986\end{array}$ & Falcy et al, ${ }^{153} 1983$ \\
\hline
\end{tabular}

Table IV. Recommended target plasma concentration ranges for antidepressant drugs and levels of recommendation for routine monitoring. ${ }^{11}$ Therapeutic ranges indicate trough concentrations of drugs in serum or plasma of patients under steady-state medication. Level of recommendation: 1. Strongly recommended (for lithium TDM should be a standard of care): established therapeutic range; 2 . Recommended: suggested therapeutic ranges obtained from plasma concentrations at therapeutically effective doses (fixed dose studies); 3. Useful: suggested therapeutic ranges are plasma concentrations at therapeutically effective doses obtained from steady-state pharmacokinetic studies; 4. Probably useful: suggested therapeutic ranges from steady-state pharmacokinetic studies at therapeutically effective doses; 5 . Not recommended. SPC, Summary of Product Characteristics. 


\section{Pharmacological aspects}

However, the data presented in Table III are insufficient to allow levels of recommendations 1 or 2, as it does not include studies on the plasma concentration-clinical effectiveness relationship. Therefore, the literature had to be reexamined to define which antidepressants may get a level 3 or 4 of recommendation for their monitoring. By consensus, a therapeutic range was then also defined for their "main" (= depression) indication (Table $I V$ ), as data for other indications (eg, anxiety disorders) are most often lacking, and some studies suggest that optimal ranges may differ, depending on the pathology. ${ }^{154}$ Antidepressants differ widely in their chemical structure and their pharmacological activity, even though most are serotonergic and/or noradrenergic. "Therapeutic windows" have been defined for most tricyclic antidepressants, and TDM is recommended to avoid intoxications, which may be lethal (Table IV).

As regards more recently introduced antidepressants, a clearcut plasma level-clinical effectiveness relationship was not demonstrated for tetracyclic antidepressants (maprotiline, mianserin, or mirtazapine), trazodone, reboxetine, the monoamine oxidase inhibitors moclobemide and tranylcypromine ${ }^{133}$ and SSRIs. ${ }^{21,155,156}$ However, TDM of SSRIs was shown to be cost-effective, as it helps to use minimum effective doses. ${ }^{114}$ Therefore, data on the plasma concentrations at therapeutic doses may be clinically useful for these drugs (Table III), in situations of noncompliance, nonresponse, adverse effects, or intoxication.

\section{Specific indications for TDM in psychiatry}

Therapeutic windows should be interpreted in the context of the clinical situation, before the decision to change treatment strategy is taken. As an example, low levels may be sufficient for the antidepressant doxepin, if it is used to obtain sedation. ${ }^{95}$

Interestingly, despite the increasing use of generics, there are few data available that demonstrate unambiguously the occurrence of pharmacokinetic problems after switching from an original preparation to a generic form (and vice versa). ${ }^{157-160} \mathrm{TDM}$ is a general indication for the administration of psychotropic drugs in children and adolescents because psychopharmacotherapy of children and adolescents differs from that of adults (Gerlach et al, in press): (i) There are differences in the pharmacokinetic behavior of drugs used in dependence on the stage of development; it is therefore not appropriate to use dosages recommended for adults. (ii) Many drugs are not approved for use in children and adolescents; the consequence is that the criteria for efficacy and safety, guaranteed for the use in adults, are not given for administration in children and adolescents. There is, however, a need to carry out standardized studies to find therapeutic ranges of plasma concentrations for children and adolescents.

In these patients, but also in elderly subjects, TDM may help distinguish between pharmacokinetic and pharmacodynamic factors in the occurrence of adverse effects. Consequently, TDM also represents a useful tool in situations of pharmacovigilance programs.

Antidepressants should be monitored in the blood of pregnant or lactating women in order to minimize drug exposure of the fetus or newborn infant. .61-165 $^{\text {" }}$

Investigations on the "therapeutic window" of patients should not only be included in phase IV studies. If possible, they should also be carried out in phase III studies, in relationship with clinical ratings, in order to propose TDM with the introduction of the new drug. As stated in the document published by the European Agency for the Evaluation of Medicinal Products, ${ }^{166}$ an established concentration-response relationship is the basis to forecast the chance of toxicity due to pharmacokinetic differences, drug-disease, or drug-drug interactions.

\section{Pharmacogenetic tests in addition to TDM}

There is increasing evidence for an advantage to combine pharmacogenetic tests with TDM..$^{18,39,44,167}$ However, pharmacogenetic tests alone have limited value, as environmental factors also regulate drug metabolism. ${ }^{168}$ Some of the most important indications for phenotyping and/or genotyping (in combination with TDM) are the following. ${ }^{51,168}$

- The metabolism of the medication (or its active metabolite) is governed to a significant extent by the enzyme, which is considered to be phenotyped or genotyped.

- The patient is treated with a substrate whose metabolism shows a wide interindividual variability, as demonstrated by TDM.

- A drug is characterized by a low therapeutic index, ie, risk of toxicity in the case of a genetically impaired metabolism or, on the other hand, risk of nonresponse due to an ultrarapid metabolism and the inability to reach therapeutic drug levels.

- The patient presents unusual plasma concentrations of the drug or its metabolite(s), and genetic factors are suspected to be responsible. 
- The patient suffers from a chronic illness, which requires life-long treatment.

As outlined above, both phenotyping and genotyping are recommended in some circumstances, as a "traitmarker" and a "state-marker." Currently, data obtained by TDM represent a "state-marker."

\section{Practical aspects of TDM}

Previous studies suggest that the "compliance" of the treating physician needs to be improved, as many requests or indications for TDM were inappropriate. ${ }^{169}$ Moreover, clinicians frequently do not follow the recommendations given by the laboratory to adjust the treatment. ${ }^{73}$ Therefore, some practical recommendations are summarized (see reference 11 for a comprehensive presentation) for the optimal use of TDM, as illustrated in Figure 1.

\section{Recommendations for the treating physician}

\section{Preparation of TDM}

Some patients may particularly benefit from TDM: an antidepressant drug should then be recommended for which TDM is available, either to minimize adverse effects or optimize its clinical efficacy. A well-defined "therapeutic window" for this drug (Table IV) or at least known plasma concentration ranges for clinical doses (Table II) should be available.

Blood should be collected for TDM in steady-state conditions, ie, at least 5 drug half-lives after changes in dose and during the terminal $\beta$-elimination phase. Generally, the appropriate sampling time for most antidepressants (except for fluoxetine) is 1 week after stable daily dosing and immediately before ingestion of the morning dose, ie, about 12 to $16 \mathrm{~h}$ (or $24 \mathrm{~h}$ if the drug is given once daily) after the last medication. It should be considered that both after a modification of the dose and after prescription of a comedication, which may inhibit or enhance the metabolism of the drug to be measured, steady-state conditions are reached again only after a few days. TDM should then be delayed, in case unexpected side effects are observed.

Most antidepressants are stable in serum or plasma for at least $24 \mathrm{~h}^{170}$ and can therefore be sent to the laboratory at room temperature. It is mandatory to consider technical recommendations given by the laboratory: choice of anti- coagulant (plasma, serum), sample volume and its labeling, conditions for mailing, influence of light, and temperature. Information on comedication may help the laboratory to avoid analytical problems (interferences with other drugs). It is strongly recommended to fill out the request forms adequately and completely (diagnosis, comorbidities, comedications, treatment duration, doses, sex and age of the patient, and reasons for the request), in order to allow interpretation of the result by clinical pharmacologists. Some of these data may also represent important information for the laboratory to judge plausibility of the result.

\section{Critical appreciation of the results}

A pharmacological treatment should be guided by sound clinical judgment. TDM has to be considered as an additional and useful tool for optimizing therapy.

Analytical methods used in the laboratories may differ in their quality. The physician should be aware that some drug levels are not accurately measured, even though most laboratories have introduced a program to measure quality. Indeed, worldwide external quality-control programs show considerable variability between laboratories in the results of analysis of control samples. The physician may obtain discrepant results when a drug was monitored several times in a patient, but analyzed in different laboratories. When comparisons of TDM values obtained from different laboratories are carried out, the clinician should take into account the units $(\mathrm{ng} / \mathrm{mL}$, $\mu \mathrm{g} / \mathrm{L}, \mu \mathrm{mol} / \mathrm{L}, \mathrm{nmol} / \mathrm{L})$ in which the results of the analysis are expressed.

Low plasma drug concentrations suggest either irregular intake of the drug or ultrarapid metabolism, and in this situation, a pharmacogenetic test may be indicated. In the first case, TDM should be repeated in order to verify compliance. These examples show that it may be advantageous for the clinician to collaborate with a TDM laboratory that offers pharmacological consultation.

\section{TDM interpretation and treatment of patients}

A TDM result represents a guide to adjust the treatment of the individual patient, but expert interpretation and adequate use of this pharmacokinetic data are mandatory for an optimal clinical benefit. Reporting of results and inclusion of dose recommendations and other com- 


\section{Pharmacological aspects}

ments by the laboratory must be guided by the best available evidence. However, the laboratory has only limited knowledge of the clinical context. The physician should also take into consideration whether the "reference plasma concentrations range" reflects only "drug plasma concentrations at clinically relevant doses" (Table III) or whether they are "therapeutic ranges" (Table IV). Information on the level of recommendation for TDM of the particular drug may also help evaluate the clinical significance of the result (Table IV). If the plasma concentration of the drug is within the therapeutic range, an adaptation of the dose is, of course, only recommended when clinical reasons, such as adverse effects or nonresponse, clearly justify such a decision. When the advice given on the TDM report is not followed, the reason for such a decision should be carefully documented.

\section{Recommendations for the laboratory}

\section{Analytical procedures}

The concentrations of antidepressants are generally low, in the $\mathrm{ng} / \mathrm{mL}$ range, and many patients are comedicated with various, potentially interfering drugs. The methods should be adapted to this situation by precision (coefficient of variation $<15 \%$ ), accurateness (deviation from nominal value $<15 \%$ ), and robustness. ${ }^{5}$ Each assay needs to be validated, documented, and regularly assessed for linearity, selectivity, accuracy, precision, recovery, and sensitivity (limits of detection [LOD] and quantification [LOQ]). Internal and external quality control procedures are mandatory to ensure maximal quality of TDM. If quality controls are outside the expected range, the reason underlying the outlier needs to be clarified and documented..$^{64.66}$

Where indicated the laboratory should analyze both the drug and its active metabolite(s) (Tables II and III). Moreover, the analysis of (active and inactive) metabolites represents an additional tool to verify compliance of patients.

\section{Reporting of results}

In addition to the result, the appropriate target range should be communicated to the physician (Tables II and III), using, of course, the same units (either mass or molar units). The LOD, or preferentially the LOQ, should be indicated in situations when plasma drug concentrations are below these values. The results should be available for clinical interpretation within a clinically meaningful time, especially in case of suspected intoxications. An interpretation and clinical and pharmacological advice should be provided with every report. Therefore, it is advantageous for the clinician to choose a laboratory that offers this service.

Plasma concentrations must be interpreted in the light of sound clinical judgment. Most frequently, recommendations on dose changes are given, and in a situation of drug concentrations above the recommended range, rapidity of communication may enhance successful intervention in patients at risk of toxicity. The physician will also appreciate comments related to genetic polymorphisms, risk for pharmacokinetic interactions in situations, and pharmacokinetic properties of the drug when given to elderly patients or patients with hepatic or renal insufficiency.

In situations where drug concentrations are particularly low, it is often not clear whether the patient is an UM or whether he or she is noncompliant in that the drug intake is irregular. The analysis of a second plasma sample may help verify compliance but, depending on the result, a pharmacogenetic test should be carried out.

Clearly a PM (CYP 2D6) status should not automatically result in interruption of a treatment, ${ }^{18,171}$ but the dose should be adapted using clinical judgment and TDM.

\section{Conclusion}

TDM is a valuable approach to optimize both shortterm and lifelong treatment of psychiatric patients with antidepressants, ${ }^{172}$ and a combination of TDM with pharmacogenetic tests will be increasingly useful, particularly because in near future, pharmacogenetic tests regarding pharmacodynamic parameters will also be clinically relevant. ${ }^{173}$ Many data on plasma concentrations of psychotropic drugs and the plasma concentration-clinical effectiveness relationship have accumulated over the past few years, and encouraged this interdisciplinary collaboration of specialists who brought about this consensus on TDM. ${ }^{11}$ Hopefully, it will help to use TDM optimally from a scientific, clinical, and economic point of view. 


\section{Las pautas de consenso del grupo de expertos AGNP-TDM: foco en el monitoreo terapéutico de los antidepresivos}

El monitoreo terapéutico de fármacos (TDM), que incluye los antidepresivos entre los psicofármacos, se ha introducido extensamente para optimizar la farmacoterapia en los pacientes psiquiátricos. El grupo interdisciplinario de TDM del Arbeitsgemeinschaft für Neuropsychopharmakologie und Pharmakopsychiatrie (AGNP) ha trabajado en pautas de consenso con el objetivo de entregar a los psiquiatras y a los laboratorios de TDM una herramienta que permita optimizar el empleo del TDM. Se definieron cinco niveles de recomendación basados en la investigación relacionada con el monitoreo de rutina de concentraciones plasmáticas de fármacos: (1) altamente recomendado, (2) recomendado, (3) útil, (4) probablemente útil y (5) no recomendado. Además se presentó una lista de indicaciones que justifican el uso del TDM, como por ejemplo, el control de la adherencia, la falta de respuesta clínica o los efectos adversos a dosis recomendadas, las interacciones de fármacos, los programas de farmacovigilancia, la presencia de alguna particularidad genética en relación con el metabolismo de los fármacos y los pacientes infanto-juveniles y ancianos. Para algunos fármacos faltan estudios en rangos terapéuticos. De acuerdo con estudios farmacocinéticos reportados en la literatura, se presentan los rangos objetivos para concentraciones plasmáticas clínicamente relevantes de la mayoría de los fármacos. Para muchos antidepresivos un completo análisis de la literatura de los estudios que abordan la relación concentración plasmática-eficacia clínica ha permitido la inclusión de rangos terapéuticos de concentraciones plasmáticas. Además, se han realizado recomendaciones relacionadas con la combinación de pruebas farmacogenéticas (fenotipo o genotipo) con el TDM. Finalmente, se han entregado instrucciones prácticas para los profesionales de los laboratorios y los psiquiatras tratantes de cómo utilizar el TDM: preparación del TDM, análisis de fármacos, informe e interpretación de resultados y un adecuado uso de la información para el tratamiento del paciente. EI TDM es un proceso complejo que requiere de una óptima coordinación interdisciplinaria de un procedimiento que involucra pacientes, psiquiatras tratantes, farmacólogos clínicos y especialistas en laboratorio clínico. Esta pauta de consenso debiera ser útil para optimizar el TDM de los antidepresivos.

\section{REFERENCES}

1. American Psychiatric Association Practice Guidelines. Practice guideline for the treatment of patients with major depressive disorder (revision). Am J Psychiatry. 2000;157(suppl 4):1-45

2. Bauer M, Whybrow PC, Angst J, Versiani M, Möller HJ. World Federation of Societies of Biological Psychiatry (WFSBP) guidelines for biological treatment of unipolar depressive disorders. Part 1: acute and continuation treatment of major depressive disorder. World J Biol Psychiatry. 2002;3:5-43.

3. Donoghue J, Taylor DM. Suboptimal use of antidepressants in the treatment of depression. CNS Drugs. 2000;13:365-383.

4. Hirschfeld RMA, Montgomery SA, Aguglia E, et al. Partial response and nonresponse to antidepressant therapy: current approaches and treatment options. J Clin Psychiatry. 2002;63:826-837.

5. Olver JS, Burrows GD, Norman TR. Third-generation antidepressantsdo they offer advantages over the SSRIs? CNS Drugs. 2001;15:941-954.

6. Nelson JC. Augmentation strategies in depression 2000. J Clin Psychiatry. 2000;61(suppl 2):13-19.

7. Zullino $D$, Baumann P. Lithium augmentation in depressive patients not responding to selective serotonin reuptake inhibitors. Pharmacopsychiatry. 2001;34:119-127.

8. Stimpson N, Agrawal N, Lewis G. Randomised controlled trials investigating pharmacological and psychological interventions for treatmentrefractory depression. Br J Psychiatry. 2002;181:284-294.
9. Thase ME. The need for clinically relevant research on treatment-resistant depression. J Clin Psychiatry. 2001;62:221-224.

10. Michelson D, Allen AJ, Busner J, et al. Once-daily atomoxetine treatment for children and adolescents with attention deficit hyperactivity disorder: a randomized, placebo-controlled study. Am J Psychiatry. 2002;159:1896-1901.

11. Baumann P, Hiemke C, Ulrich $S$, et al. The AGNP-TDM expert group consensus guidelines: therapeutic drug monitoring in psychiatry. Pharmacopsychiatry. 2004;37:243-265.

12. Åsberg $M$, Cronholm B, Sjöqvist $F$, Tuck D. Relationship between plasma level and therapeutic effect of nortriptyline. BMJ. 1971;3:331-334.

13. Bertilsson L, Mellström B, Sjöqvist F, Mårtensson B, Åsberg M. Slow hydroxylation of nortriptyline and concomitant poor debrisoquine hydroxylation: clinical implications. Lancet. 1981;i:560-561.

14. Task Force. Tricyclic antidepressants—-blood level measurements and clinical outcome: an APA Task Force report. Am J Psychiatry. 1985;142:155162.

15. Orsulak PJ. Therapeutic monitoring of antidepressant drugs-guidelines updated. Ther Drug Monit. 1989;11:497-507.

16. Linder MW, Keck PE, Jr. Standards of laboratory practice: antidepressant drug monitoring. Clin Chem. 1998;44:1073-1084.

17. Laux G, Riederer P (eds). Plasmaspiegel-bestimmung von Psychopharmaka: Therapeutisches Drug - Monitoring Versuch einer ersten Standortbestimmung. Stuttgart, Germany: Wissenschaftliche Verlagsgesellschaft mbH; 1992. 


\section{Pharmacological aspects}

Recommandations du groupe d'experts AGNP-TDM: Le monitoring à but thérapeutique des antidépresseurs

Le dosage plasmatique de médicaments psychotropes dans un but thérapeutique (therapeutic drug monitoring (TDM)) y compris des antidépresseurs a été largement introduit pour optimiser la pharmacothérapie de patients psychiatriques. Le groupe interdisciplinaire AGNP-TDM (Arbeitsgemeinschaft für Neuropsychopharmakologie und Phamakopsychiatrie; Association de Neuro-psycho-pharmacologie et de Pharmacopsychiatrie) a élaboré des recommandations dans le but de procurer aux psychiatres et aux laboratoires TDM un outil pour optimiser l'utilisation du TDM. Basés sur des résultats obtenus par la recherche, cinq niveaux de recommandations ont été définis par rapport au monitoring de routine des taux plasmatiques de médicaments : 1. fortement recommandé, 2. recommandé, 3. utile, 4. probablement utile, 5. pas recommandé. De plus une liste d'indications qui justifient l'utilisation des TDM est présentée, par exemple : contrôle de l'observance, absence de réponse clinique ou effets secondaires à des doses généralement recommandées, interactions médicamenteuses, programme de pharmacovigilance, présence d'une particularité génétique concernant le métabolisme de médicaments, enfants, adolescents et patients âgés. Pour quelques médicaments, des études sur les marges thérapeutiques manquent, mais des marges cibles pour des concentrations plasmatiques cliniquement significatives sont présentées pour la plupart des médicaments, basées sur des études pharmacocinétiques rapportées dans la littérature. Pour beaucoup d'antidépresseurs, une analyse complète de la littérature sur les études qui traitent de la relation concentration plasmatique - efficacité clinique a permis de présenter des marges thérapeutiques de concentrations plasmatiques. En outre, des recommandations sont données par rapport à la combinaison de tests pharmacogénétiques (phénotypage ou génotypage) avec le TDM. Finalement, des instructions pratiques sont données aux techniciens responsables de laboratoires et aux médecins traitants qui utilisent le TDM : préparation du TDM, analyses de médicaments, communication et interprétation du résultat et utilisation adéquate de l'information pour le traitement du patient. Le TDM est un processus qui nécessite une coordination interdisciplinaire optimale d'une procédure qui implique des patients, des médecins traitants, des pharmacologues cliniques et des spécialistes du laboratoire clinique. Ce « Consensus guideline " (recommandations) devrait être utile pour optimiser le TDM d'antidépresseurs.

18. Kirchheiner J, Brøsen K, Dahl ML, et al. CYP2D6 and CYP2C19 genotypebased dose recommendations for antidepressants: a first step towards subpopulation-specific dosages. Acta Psychiatr Scand. 2001;104:173-192.

19. Dahl ML. Cytochrome P450 phenotyping/genotyping in patients receiving antipsychotics: useful aid to prescribing? Clin Pharmacokinet. 2002;41:453470 .

20. Ulrich S, Wurthmann C, Brosz M, Meyer FP. The relationship between serum concentration and therapeutic effect of haloperidol in patients with acute schizophrenia. Clin Pharmacokinet. 1998;34:227-263.

21. Hiemke $C$, Härtter $S$. Pharmacokinetics of selective serotonin reuptake inhibitors. Pharmacol Ther. 2000;85:11-28.

22. Greenblatt DJ, Von Moltke LL, Harmatz JS, Shader RI. Human cytochromes and some newer antidepressants: kinetics, metabolism, and drug interactions. J Clin Psychopharmacol. 1999;19(suppl 1):23S-35S.

23. Nemeroff $C B$, DeVane CL, Pollock BG. Newer antidepressants and the cytochrome P450 system. Am J Psychiatry. 1996;153:311-320.

24. Meyer UA, Amrein R, Balant LP, et al. Antidepressants and drug-metabolizing enzymes - expert group report. Acta Psychiatr Scand. 1996;93:71-79. 25. Rudorfer MV, Potter WZ. Metabolism of tricyclic antidepressants. Cell Mol Neurobiol. 1999;19:373-409.

26. Breyer-Pfaff U, Gaertner HJ, Baumann P. AntidepressivaPharmakologie, therapeutischer Einsatz und Klinik der Depressionen. 2nd ed. Stuttgart, Germany: Wissenschaftliche Verlagsgesellschaft mbH; 2005. 27. Marzo A, Balant LP. Investigation of xenobiotic metabolism by CYP2D6 and CYP2C19: importance of enantioselective analytical methods. $J$ Chromatogr B Biomed Appl. 1996;678:73-92.
28. Baumann P, Zullino DF, Eap CB. Enantiomers' potential in psychopharmacology - a critical analysis with special emphasis on the antidepressant escitalopram. Eur Neuropsychopharmacol. 2002;12:433-444.

29. Testa B. Drug metabolism. In: Wolff ME, ed. Burger's Medicinal Chemistry and Drug Discovery. 5th ed. New York, NY: John Wiley and Sons; 1995:129-180.

30. Liston HL, Markowitz JS, DeVane CL. Drug glucuronidation in clinical psychopharmacology. J Clin Psychopharmacol. 2001;21:500-515.

31. Burchell B. Transformation reactions: glucuronidation. In: Woolf TF, ed. Handbook of Drug Metabolism. New York, NY: Marcel Dekker; 1999:153-173. 32. Penzak SR, Hon YY, Lawhorn WD, Shirley KL, Spratlin V, Jann MW. Influence of ritonavir on olanzapine pharmacokinetics in healthy volunteers. J Clin Psychopharmacol. 2002;22:366-370.

33. Caccia S, Garattini S. Formation of active metabolites of psychotropic drugs: an updated review of their significance. Clin Pharmacokinet. 1990;18:434-459.

34. Eadie MJ. Formation of active metabolites of anticonvulsant drugs-a review of their pharmacokinetic and therapeutic significance. Clin Pharmacokinet. 1991;21:27-41.

35. Rudorfer MV, Potter WZ. The role of metabolites of antidepressants in the treatment of depression. CNS Drugs. 1997;7:273-312.

36. Bertilsson L, Dahl ML, Dalen $P$, Al Shurbaji A. Molecular genetics of CYP 2D6: clinical relevance with focus on psychotropic drugs. $\mathrm{Br} J$ Clin Pharmacol. 2002;53:111-122.

37. Cascorbi I. Pharmacogenetics of cytochrome P4502D6: genetic background and clinical implication. Eur J Clin Invest. 2003;33(suppl 2):17-22. 
38. Murray M. P450 Enzymes. Inhibition mechanisms, genetic regulation and effects of liver disease. Clin Pharmacokinet. 1992;23:132-146.

39. Dahl ML, Sjöqvist F. Pharmacogenetic methods as a complement to therapeutic monitoring of antidepressants and neuroleptics. Ther Drug Monit. 2000;22:114-117

40. Kirchheiner J, Nickchen K, Bauer M, et al. Pharmacogenetics of antidepressants and antipsychotics: the contribution of allelic variations to the phenotype of drug response. Mol Psychiatry. 2004;9:442-473.

41. Vasiliou V, Pappa A, Petersen DR. Role of aldehyde dehydrogenases in endogenous and xenobiotic metabolism. Chem Biol Interact. 2000;129:1-19. 42. Coutts RT, Urichuk LJ. Polymorphic cytochromes P450 and drugs used in psychiatry. Cell Mol Neurobiol. 1999;19:325-354.

43. Benet LZ, Kroetz DL, Sheiner LB. Pharmacokinetics the dynamics of drug absorption, distribution, and elimination. In: Hardman JG, Limbird LE, eds. Goodman \& Gilman's The Pharmacological Basis of Therapeutics. 9th ed. New York, NY: McGraw-Hill; 1996:3-27.

44. Scordo MG, Spina E. Cytochrome P450 polymorphisms and response to antipsychotic therapy. Pharmacogenomics. 2002;3:201-218.

45. Kuehl $P$, Zhang J, Lin $Y$, et al. Sequence diversity in CYP3A promoters and characterization of the genetic basis of polymorphic CYP3A5 expression. Nat Genet. 2001;27:383-391.

46. Nakajima M, Yokoi T, Mizutani M, Kinoshita M, Funayama M, Kamataki T. Genetic polymorphism in the 5'-flanking region of human CYP1A2 gene: effect on the CYP1A2 inducibility in humans. J Biochem. 1999:125:803-808. 47. Sachse C, Brockmöller J, Bauer S, Roots I. Functional significance of a C_A polymorphism in intron I of the cytochrome P450 CYP 1 A2 gene tested with caffeine. Br J Clin Pharmacol. 1999:47:445-449.

48. Brøsen K. Drug-metabolizing enzymes and therapeutic drug monitoring in psychiatry. Ther Drug Monit. 1996;18:393-396.

49. DeVane CL. Pharmacogenetics and drug metabolism of newer antidepressant agents. J Clin Psychiatry. 1994;55(suppl 12):38-45.

50. Otani K, Aoshima T. Pharmacogenetics of classical and new antipsychotic drugs. Ther Drug Monit. 2000;22:118-121.

51. Steimer W, Potter JM. Pharmacogenetic screening and therapeutic drugs. Clin Chim Acta. 2002;315:137-155.

52. Kirchheiner J, Bertilsson L, Bruus H, Wolff A, Roots I, Bauer M. Individualized medicine-implementation of pharmacogenetic diagnostics in antidepressant drug treatment of major depressive disorders. Pharmacopsychiatry. 2003;36(suppl 3):S235-S243.

53. Eap $C B$, Jaquenoud Sirot $E$, Baumann $P$. Therapeutic monitoring of antidepressants in era of pharmacogenetics studies. Ther Drug Monit. 2004;26:152-155

54. De Leon J. Glucuronidation enzymes, genes and psychiatry. Int J Neuropsychopharmacol. 2003;6:57-72.

55. Marzolini C, Paus E, Buclin T, Kim RB. Polymorphisms in human MDR1 (P-glycoprotein): recent advances and clinical relevance. Clin Pharmacol Ther. 2004;75:13-33.

56. Uhr M, Grauer MT. abcb1ab P-glycoprotein is involved in the uptake of citalopram and trimipramine into the brain of mice. J Psychiatr Res. 2003;37:179-185

57. Uhr M, Steckler T, Yassouridis A, Holsboer F. Penetration of amitriptyline, but not of fluoxetine, into brain is enhanced in mice with blood-brain barrier deficiency due to Mdr1a P-glycoprotein gene disruption. Neuropsychopharmacology. 2000;22:380-387.

58. Perry PJ. The relationship between antidepressant response and tricyclic antidepressant plasma concentrations. A retrospective analysis of the literature using logistic regression analysis. Clin Pharmacokinet. 1987;13:381-392. 59. Perry PJ, Zeilmann C, Arndt S. Tricyclic antidepressant concentrations in plasma: an estimate of their sensitivity and specificity as a predictor of response. J Clin Psychopharmacol. 1994;14:230-240.

60 . Ulrich S, Läuter J. Comprehensive survey of the relationship between serum concentration and therapeutic effect of amitriptyline in depression. Clin Pharmacokinet. 2002;41:853-876.

61. Balant-Gorgia EA, Balant LP. Therapeutic drug monitoring-relevance during the drug treatment of psychiatric disorders. CNS Drugs. 1995;4:432453.

62. Eilers R. Therapeutic drug monitoring for the treatment of psychiatric disorders — clinical use and cost effectiveness. Clin Pharmacokinet. 1995;29:442-450.
63. Green M. A practical guide to analytical method validation. Anal Chem News Features. 1996:305-309.

64. Buick AR, Doig MV, Jeal SC, Land GS, Mcdowall RD. Method validation in the bioanalytical laboratory. J Pharm Biomed Anal. 1990;8:629-637.

65. Shah VP, Midha KK, Dighe S, et al. Analytical methods validationbioavailability, bioequivalence and pharmacokinetic studies. Pharm Res. 1992;9:588-592.

66. Causon R. Validation of chromatographic methods in biomedical analysis viewpoint and discussion. J Chromatogr B. 1997;689:175-180.

67. de la Torre R, Ortuño J, Pascual JA, Gonzalez S, Ballesta J. Quantitative determination of tricyclic antidepressants and their metabolites in plasma by solid-phase extraction (bond-elute TCA) and separation by capillary gas chromatography with nitrogen-phosphorus detection. Ther Drug Monit. 1998;20:340-346.

68. Banger M, Hermes B, Härtter S, Hiemke C. Monitoring serum concentrations of clomipramine and metabolites: fluorescence polarization immunoassay versus high performance liquid chromatography. Pharmacopsychiatry. 1997;30:128-132.

69. Kollroser M, Schober C. Simultaneous determination of seven tricyclic antidepressant drugs in human plasma by direct-injection HPLC-APCI-MSMS with an ion trap detector. Ther Drug Monit. 2002;24:537-544.

70. Veuthey JL, Souverain S, Rudaz S. Column-switching procedures for the analysis of drugs in biologic samples. Ther Drug Monit. 2004;26:161-166.

71. Simmons SA, Perry PJ, Rickert ED, Browne JL. Cost-benefit analysis of prospective pharmocokinetic dosing of nortriptyline in depressed inpatients. J Affect Disord. 1985;8:47-53.

72. Lundmark J, Bengtsson F, Nordin C, Reis M, Walinder J. Therapeutic drug monitoring of selective serotonin reuptake inhibitors influences clinical dosing strategies and reduces drug costs in depressed elderly patients. Acta Psychiatr Scand. 2000;101:354-359.

73. Müller MJ, Dragicevic A, Fric M, et al. Therapeutic drug monitoring of tricyclic antidepressants: how does it work under clinical conditions? Pharmacopsychiatry. 2003;36:98-104.

74. Chen S, Chou WH, Blouin RA, et al. The cytochrome P450 2D6 (CYP2D6) enzyme polymorphism: screening costs and influence on clinical outcomes in psychiatry. Clin Pharmacol Ther. 1996;60:522-534

75. Chou WH, Yan FX, Barnhill J, et al. Extension of a pilot study: impact from the cytochrome P450 2D6 polymorphism on outcome and costs associated with severe mental illness. J Clin Psychopharmacol. 2000;20:246-251.

76. Veenstra DL, Higashi MK. Assessing the cost-effectiveness of pharmacogenomics. AAPS Pharmsci. 2000;2:1-11.

77. Baumann $\mathrm{P}$, Jonzier-Perey $\mathrm{M}$, Koeb L, Le PK, Tinguely D, Schöpf J. Amitriptyline pharmacokinetics and clinical response. I. Free and total plasma amitriptyline and nortriptyline. Int Clin Psychopharmacol. 1986;1:89101.

78. Jungkunz G, Kuss HJ. On the relationship of nortriptyline: amitriptyline ratio to clinical improvement of amitriptyline-treated depressive patients. Pharmakopsychiatr Neuropsychopharmakol. 1980;13:111-116.

79. Breyer-Pfaff U, Gaertner HJ, Kreuter F, Scharek G, Brinkschulte M, Wiatr R. Antidepressive effect and pharmacokinetics of amitriptyline with consideration of unbound drug and 10-hydroxynortriptyline plasma levels. Psychopharmacology. 1982;76:240-244.

80. Breyer-Pfaff U, Gaertner HJ, Giedke H. Plasma levels, psychophysiological variables, and clinical response to amitriptyline. Psychiatry Res. 1982:6:223-234.

81. Kupfer DJ, Hanin I, Spiker DG, Grau T, Coble P. Amitriptyline plasma levels and clinical response in primary depression. Clin Pharmacol Ther. 1977;22:904-911.

82. Baumann P, Nil R, Souche A, et al. A double-blind, placebo-controlled study of citalopram with and without lithium in the treatment of therapyresistant depressive patients: a clinical, pharmacokinetic, and pharmacogenetic investigation. J Clin Psychopharmacol. 1996;16:307-314.

83. Baumann P, Nil R, Bertschy G, et al. A double-blind double-dummy study of citalopram comparing infusion versus oral administration. J Affect Disord. 1998;49:203-210

84. Kramer Nielsen K, Brøsen K, Gram LF, et al. Steady-state plasma levels of clomipramine and its metabolites: impact of the sparteine/debrisoquine oxidation polymorphism. Eur J Clin Pharmacol. 1992;43:405-411. 


\section{Pharmacological aspects}

85. Danish University Antidepressant Group. Clomipramine dose-effect study in patients with depression: clinical end points and pharmacokinetics. Clin Pharmacol Ther. 1999;66:152-165.

86. Müller-Oerlinghausen B, Fahndrich $\mathrm{E}$. The relationship between pharmacokinetic data and the clinical response in patients treated with maprotiline or clomipramine by intravenous infusion. Pharmacopsychiatry. 2002;18:100-101.

87. Burch JE, Raddats MA. Time course of plasma drug levels during once-daily oral administration of clomipramine. Psychopharmacology. 1982;77:344-347. 88. Friedel RO, Veith RC, Bloom V, Bielski RJ. Desipramine plasma levels and cliical response in depressed outpatients. Commun Psychopharmacol. 1979;3:81-87.

89. Amsterdam JD, Brunswick DJ, Winokur A, Rickels K. Desipramine and 2 hydroxydesipramine plasma levels in endogenous depressed patients. Arch Gen Psychiatry. 1985:42:361-364.

90. Nelson JC, Jatlow PI, Mazure C. Desipramine plasma levels and response in elderly melancholic patients. J Clin Psychopharmacol. 1985;5:217-220.

91. Maguire KP, Norman TR, Burrows GD, Davies B. Blood and plasma concentrations of dothiepin and its major metabolites and clinical response. $J$ Affect Disord. 1982;4:41-48.

92. Ilett KF, Blythe TH, Hackett LP, Ong RTT, Tannenbaum DA, Clarke TMF. Plasma concentrations of dothiepin and its metabolites are not correlated with clinical efficacy in major depressive illness. Ther Drug Monit 1993;15:351-357

93. Adler L, Hajak G, Lehmann K, et al. On the problems of switching from intravenous to oral administration in drug treatment of endogenous depression-a placebo-controlled double-blind trial with doxepin Pharmacopsychiatry. 1997;30:62-69.

94. Deuschle M, Härtter S, Hiemke C, Standhardt H, Heuser I. Doxepin and its metabolites in plasma and cerebrospinal fluid in depressed patients. Psychopharmacology. 1997;131:19-22.

95. Leucht S, Steimer W, Kreuz S, Abraham D, Orsulak PJ, Kissling W. Doxepin plasma concentrations: is there really a therapeutic range? J Clin Psychopharmacol. 2001;21:432-439.

96. Bondolfi G, Chautems C, Rochat B, Bertschy G, Baumann P. Non-response to citalopram in depressive patients: pharmacokinetic and clinical consequences of a fluvoxamine augmentation. Psychopharmacology. 1996;128:421 425.

97. Bondolfi G, Lissner C, Kosel M, Eap CB, Baumann P. Fluoxetine augmentation in citalopram non-responders: pharmacokinetic and clinical consequences. Int J Neuropsychopharmacol. 2000;3:55-60.

98. Lundmark J, Reis M, Bengtsson F. Serum concentrations of fluoxetine in the clinical treatment setting. Ther Drug Monit. 2001;23:139-147.

99. Amsterdam JD, Fawcett J, Quitkin FM, et al. Fluoxetine and norfluoxetine plasma concentrations in major depression: a multicenter study. Am Psychiatry. 1997;154:963-969.

100. Härtter S, Wetzel H, Hammes E, Torkzadeh M, Hiemke C. Nonlinear pharmacokinetics of fluvoxamine and gender differences. Ther Drug Monit. 1998;20:446-449.

101. Kasper S, Dötsch M, Kick H, Vieira A, Möller HJ. Plasma concentrations of fluvoxamine and maprotiline in major depression: implications on ther apeutic efficacy and side effects. Eur Neuropsychopharmacol. 1993;3:13-21.

102. Gerstenberg G, Aoshima T, Fukasawa T, et al. Relationship between clinical effects of fluvoxamine and the steady-state plasma concentrations of fluvoxamine and its major metabolite fluvoxamino acid in Japanese depressed patients. Psychopharmacology. 2003;167:443-448.

103. Reisby N, Gram LF, Bech P, et al. Imipramine: clinical effects and pharmacokinetic variability. Psychopharmacology. 1977;54:263-272.

104. Gabris G, Baumann $P$, Jonzier-Perey $M$, Bosshart $P$, Woggon B, Küpfer A. $N$-Methylation of maprotiline in debrisoquine/mephenitoin-phenotyped depressive patients. Biochem Pharmacol. 1985;34:409-410.

105. Otani K, Kaneko S, Sasa H, Kondo T, Fukushima Y. Is there a therapeutic window for plasma concentration of mianserin plus desmethylmianserin? Hum Psychopharmacol Clin Exp. 1991:6:243-248.

106. Mihara K, Otani K, Tybring G, Dahl ML, Bertilsson L, Kaneko S. The CYP 2D6 genotype and plasma concentrations of mianserin enantiomers in relation to therapeutic response to mianserin in depressed Japanese patients. J Clin Psychopharmacol. 1997;17:467-471.
107. Eap CB, Yasui N, Kaneko S, Baumann P, Powell K, Otani K. Effects of carbamazepine coadministration on plasma concentrations of the enantiomers of mianserin and of its metabolites. Ther Drug Monit. 1999;21:166170.

108. Timmer CJ, Lohmann AAM, Mink CPA. Pharmacokinetic dose-proportionality study at steady state of mirtazapine from remeron tablets. Hum Psychopharmacol. 1995;10:S97-S106.

109. Schoerlin MP, Mayersohn M, Korn A, Eggers H. Disposition kinetics of moclobemide, a monoamine oxidase A enzyme inhibitor: single and multiple dosing in normal subjects. Clin Pharmacol Ther. 1987:42:395-404.

110. Lundmark J, Thomsen IS, Fjord-Larsen T, et al. Paroxetine: pharmacokinetic and antidepressant effect in the elderly. Acta Psychiatr Scand. 1989;80(suppl 350):76-80.

111. Sindrup SH, Brøsen K, Gram LF, et al. The relationship between paroxetine and the sparteine oxidation polymorphism. Clin Pharmacol Ther. 1992;51:278-287.

112. Kaye CM, Haddock RE, Langley $P F$, et al. A review of the metabolism and pharmacokinetics of paroxetine in man. Acta Psychiatr Scand. 1989;80(suppl 350):60-75.

113. Pellizzoni C, Poggesi I, Jørgensen NP, Edwards DMF, Paus E, Strolin Benedetti M. Pharmacokinetics of reboxetine in healthy volunteers. Single against repeated oral doses and lack of enzymatic alterations. Biopharm Drug Dispos. 1996;17:623-633.

114. Lundmark J, Reis M, Bengtsson F. Therapeutic drug monitoring of sertraline: variability factors as displayed in a clinical setting. Ther Drug Monit. 2000;22:446-454.

115. Axelson DA, Perel JM, Birmaher B, et al. Sertraline pharmacokinetics and dynamics in adolescents. I Am Acad Child Adolesc Psychiatry. 2002;41:1037-1044.

116. Otani K, Tybring G, Mihara K, et al. Correlation between steady-state plasma concentrations of mianserin and trazodone in depressed patients. Eur J Clin Pharmacol. 1998:53:347-349.

117. Mihara K, Kondo T, Suzuki A, et al. Effects of genetic polymorphism of CYP 1 A2 inducibility on the steady-state plasma concentrations of trazodone and its active metabolite $\mathrm{m}$-chlorophenylpiperazine in depressed Japanese patients. Pharmacol Toxicol. 2001;88:267-270.

118. Cournoyer G, De Montigny C, Ouellette J, et al. A comparative doubleblind controlled study of trimipramine and amitriptyline in major depression: lack of correlation with 5-hydroxytryptamine reuptake blockade. J Clin Psychopharmacol. 1987; 7:385-393.

119. Eap CB, Bender S, Gastpar M, et al. Steady state plasma levels of the enantiomers of trimipramine and of its metabolites in CYP 2D6-, CYP 2C19and CYP 3A4/5-phenotyped patients. Ther Drug Monit. 2000;22:209-214.

120. Troy SM, Parker VD, Fruncillo RJ, Chiang ST. The pharmacokinetics of venlafaxine when given in a twice-daily regimen. J Clin Pharmacol. 1995;35:404-409.

121. Reis $M$, Lundmark J, Bjork $H$, Bengtsson $F$. Therapeutic drug monitoring of racemic venlafaxine and its main metabolites in an everyday clinical setting. Ther Drug Monit. 2002;24:545-553.

122. Müller-Oerlinghausen $B$, Rüther $E$. Clinical profile and serum concentration of viloxazine as compared to amitriptyline. Pharmakopsychiatr Neuropsychopharmakol. 1979;12:321-337.

123. Pedersen OL, Gram LF, Kristensen CB, et al. Overdosage of antidepressants: clinical and pharmacokinetic aspects. Eur J Clin Pharmacol. 1982;23:513521.

124. Preskorn S, Jerkovich GS. Central nervous system toxicity of tricyclic antidepressants: phenomenology, course, risk factors, and role of therapeutic drug monitoring. J Clin Psychopharmacol. 1990;10:88-95

125. Bjerkenstedt L, Flyckt L, Overø KF, Lingjaerde O. Relationship between clinical effects, serum drug concentration and serotonin uptake inhibition in depressed patients treated with citalopram. A double-blind comparison of three dose levels. Eur J Clin Pharmacol. 1985;28:553-557.

126. Leinonen E, Lepola U, Koponen H, Kinnunen I. The effect of age and concomitant treatment with other psychoactive drugs on serum concentrations of citalopram measured with a nonenantioselective method. Ther Drug Monit. 1996;18:111-117.

127. Jonasson B, Saldeen T. Citalopram in fatal poisoning cases. Forensic SCi Int. 2002;126:1-6. 
128. Gex-Fabry M, Balant-Gorgia AE, Balant LP. Clomipramine concentration as a predictor of delayed response: a naturalistic study. Eur J Clin Pharmacol. 1999;54:895-902.

129. Mavissakalian MR, Jones B, Olson S, Perel JM. Clomipramine in obsessive-compulsive disorder: clinical response and plasma levels. J Clin Psychopharmacol. 1990;10:261-268.

130. Mclntyre IM, King CV, Cordner SM, Drummer OH. Postmortem clomipramine: therapeutic or toxic concentrations? J Forensic Sci. 1994;39:486-493.

131. Rodriguez de la Torre B, Dreher J, Malevany I, et al. Serum levels and cardiovascular effects of tricyclic antidepressants and selective serotonin reuptake inhibitors in depressed patients. Ther Drug Monit. 2001;23:435-440. 132. Preskorn SH, Fast GA. Tricyclic antidepressant-induced seizures and plasma drug concentration. J Clin Psychiatry. 1992;53:160-162.

133. Goodnick PJ. Pharmacokinetic optimisation of therapy with newer antidepressants. Clin Pharmacokinet. 1994;27:307-330.

134. Montgomery SA, Montgomery DB, McAuley R, Rani SJ. Mianserin plasma levels and differential clinical response in endogenous and reactive depression. Acta Psychiatr Belg. 1978;78:798-812.

135. Isacsson $G$, Holmgren $P$, Druid $H$, Bergman $U$. The utilization of antidepressants-a key issue in the prevention of suicide: an analysis of 5281 suicides in Sweden during the period 1992-1994. Acta Psychiatr Scand. 1997;96:94-100.

136. Timmer CJ, Sitsen JMA, Delbressine LP. Clinical pharmacokinetics of mirtazapine. Clin Pharmacokinet. 2000;38:461-474.

137. Velazquez C, Carlson A, Stokes KA, Leikin JB. Relative safety of mirtazapine overdose. Vet Hum Toxicol. 2001;43:342-344.

138. Fritze J, Laux G, Sofic E, et al. Plasma moclobemide and metabolites: lack of correlation with clinical response and biogenic amines. Psychopharmacology. 1989;99:252-256.

139. Gex-Fabry M, Balant-Gorgia AE, Balant LP. Potential of concentration monitoring data for a short half-life drug: analysis of pharmacokinetic variability for moclobemide. Ther Drug Monit. 1995;17:39-46.

140. Baptista T, Teneud L, Contreras Q, et al. Lithium and body weight gain. Pharmacopsychiatry. 1995;28:35-44.

141. Åsberg M, Cronholm B, Sjöqvist F, Tuck D. Correlation of subjective side effects with plasma concentrations of nortriptyline. BMJ. 1970;4:18-21.

142. Tasker TCG, Kaye CM, Zussman BD, Link CGG. Paroxetine plasma levels: lack of correlation with efficacy or adverse events. Acta Psychiatr Scand. 1989;80(suppl 350):152-155.

143. Ohman D, Norlander B, Peterson C, Bengtsson F. Bioanalysis of racemic reboxetine and its desethylated metabolite in a therapeutic drug monitoring setting using solid phase extraction and HPLC. Ther Drug Monit. 2001;23:27-34.

144. Milner DA, Hall M, Davis GG, Brissie RM, Robinson CA. Fatal multiple drug intoxication following acute sertraline use. J Anal Toxicol. 1998;22:545548.

145. Burke MJ, Preskorn SH. Therapeutic drug monitoring of antidepressants - cost implications and relevance to clinical practice. Clin Pharmacokinet. 1999;37:147-165

146. Iwersen S, Schmoldt A. One fatal and one nonfatal intoxication with tranylcypromine. Absence of amphetamines as metabolites. J Anal Toxicol. 1996;20:301-304.

147. Monteleone P, Gnocchi G, Delrio G. Plasma trazodone concentrations and clinical response in elderly depressed patients: a preliminary study. J Clin Psychopharmacol. 1989;9:284-287.

148. Goeringer KE, Raymon L, Christian GD, Logan BK. Postmortem forensic toxicology of selective serotonin reuptake inhibitors: a review of pharmacology and report of 168 cases. J Forensic Sci. 2000;45:633-648.

149. Veefkind $A H$, Haffmans PMJ, Hoencamp E. Venlafaxine serum levels and CYP 2D6 genotype. Ther Drug Monit. 2000;22:202-208.
150. Levine B, Jenkins AJ, Queen M, Jufer R, Smialek JE. Distribution of venlafaxine in three postmortem cases. J Anal Toxicol. 1996;20:502-505.

151. Norman TR, Burrows GD, Davies BM, Maguire KP, Wurm JM. Viloxazine plasma concentrations and clinical response. J Affect Disord. 1980;2:157-164. 152. Altamura AC, Mauri MC, Guercetti G. Age, therapeutic "milieu" and clinical outcome in depressive patients treated with viloxazine: a study with plasma levels. Prog Neuropsychopharmacol Biol Psychiatry. 1986;10:67-75.

153. Falcy M, Riboulet-Delmas G, Efthymiou ML. [Acute poisoning by viloxazine chlorhydrate taken by itself]. Encéphale. 1983;9:137-144.

154. McLeod DR, Hoehn-Saric R, Porges SW, Kowalski PA, Clark CM Therapeutic effects of imipramine are counteracted by its metabolite, desipramine, in patients with generalized anxiety disorder. J Clin Psychopharmacol. 2000;20:615-621.

155. Baumann P. Pharmacokinetic-pharmacodynamic relationship of the selective serotonin reuptake inhibitors. Clin Pharmacokinet. 1996;31:444-469. 156. Rasmussen BB, Brøsen $\mathrm{K}$. Is therapeutic drug monitoring a case for optimizing clinical outcome and avoiding interactions of the selective serotonin reuptake inhibitors? Ther Drug Monit. 2000;22:143-154.

157. Baumann $P$, Kahn JM. Les médicaments génériques: quels sont les problèmes et d'où viennent-ils? Med Hyg. 2003;61:879-884.

158. Sajbel TA, Carter GW, Wiley RB. Converting patients from brand-name clozapine to generic clozapine. Ann Pharmacother. 2001;35:281-284.

159. Meredith PA. Generic drugs-therapeutic equivalence. Drug Safety. 1996;15:233-242.

160. Tse G, Thompson D, Procyshyn RM. Generic clozapine: a cost-saving alternative to brand name clozapine? PharmacoEconomics. 2003;21:1-11.

161. American Academy of Pediatrics. Use of psychoactive medication during pregnancy and possible effects on the fetus and newborn. Pediatrics. 2000;105:880-887.

162. Burt VK, Suri R, Altshuler L, Stowe Z, Hendrick VC, Muntean E. The use of psychotropic medications during breast-feeding. Am J Psychiatry. 2001;158:1001-1009.

163. Chaudron LH, Jefferson JW. Mood stabilizers during breastfeeding. A review. J Clin Psychiatry. 2001;61:79-90.

164. Llewellyn A, Stowe ZN. Psychotropic medications in lactation. J Clin Psychiatry. 1998;59(suppl 2):41-52.

165. Ernst CL, Goldberg JF. The reproductive safety profile of mood stabilizers, atypical antipsychotics, and broad-spectrum psychotropics. J Clin Psychiatry. 2002;63(suppl 4):42-55.

166. European Agency for the Evaluation of Medicinal Products (EMEA). Note for guidance on dose response information to support drug registration. 1995. Available at: www.emea.eu.int/pdfs/human/ich/037895en.pdf. Accessed 16 June 2005.

167. Bertilsson L, Dahl ML. Polymorphic drug oxidation-relevance to the treatment of psychiatric disorders. CNS Drugs. 1996;5:200-223.

168. Ensom MHH, Chang TKH, Patel P. Pharmacogenetics-the therapeutic drug monitoring of the future? Clin Pharmacokinet. 2001;40:783-802.

169. Vuille F, Amey M, Baumann P. Use of plasma level monitoring of antidepressants in clinical practice-towards an analysis of clinical utility. Pharmacopsychiatry. 1991;24:190-195.

170. Koutsilieri E, Scheller C, ter Meulen V, Riederer P. Monoamine oxidase inhibition and CNS immunodeficiency infection. Neurotoxicology. 2004;25:267-270.

171. Brockmöller J, Kirchheiner J, Schmider J, et al. The impact of the CYP 2D6 polymorphism on haloperidol pharmacokinetics and on the outcome of haloperidol treatment. Clin Pharmacol Ther. 2002;72:438-452.

172. Bengtsson F. Therapeutic drug monitoring of psychotropic drugs TDM "nouveau." Ther Drug Monit. 2004;26:145-151.

173. Bondy B, Zill P. Psychopharmacogenetics-a challenge for pharmacotherapy in psychiatry. World J Biol Psychiatry. 2001;2:178-183. 